\title{
Prognosis of split liver transplantation compared with whole liver transplantation in adult patients: single-center results under the Korean MELD score-based allocation policy
}

Sung-Min Kim, Shin Hwang, Gil-Chun Park, Gi-Won Song, Dong-Hwan Jung, Tae-Yong Ha, Chul-Soo Ahn, Deok-Bog Moon, Ki-Hun Kim, Sung-Gyu Lee

Department of Surgery, Asan Medical Center, University of Ulsan College of Medicine, Seoul, Korea

Background: Split liver transplantation (SLT) has been occasionally performed in Korea. This study compared the incidence and prognosis of SLT with whole liver transplantation (WLT) in adult patients.

Methods: Between June 2016 and November 2019, 242 adult patients underwent a total of 256 deceased donor liver transplantation (DDLT) operations. SLT was performed in seven patients (2.9\%).

Results: The mean age of SLT donors was $29.7 \pm 7.4$ years, and the mean age of recipients was $55.7 \pm 10.6$ years, with the latter having a mean model for end-stage liver disease score of $34.6 \pm 3.1$. Mean split right liver graft weight was $1228.6 \pm 149.7 \mathrm{~g}$ and mean graft-recipient weight ratio was $1.97 \pm 0.39$. Of the seven SLT recipients, Korean Network for Organ Sharing (KONOS) status was one in status 1 , one in status 2 and five in status 3 . The graft $(P=0.72)$ and patient $(P=0.84)$ survival rates were comparable in the SLT and WLT groups. Following propensity score matching, graft $(P=0.61)$ and patient $(P=0.91)$ survival rates remained comparable in the two groups. Univariate analysis showed that pretransplant ventilator support and renal replacement therapy were significantly associated with patient survival, whereas KONOS status category and primary liver diseases were not. Multivariate analysis showed that pretransplant ventilator support was an independent risk factor for patient survival.

Conclusions: Survival outcomes were similar in adult SLT and WLT recipients, probably due to selection of high-quality grafts and low-risk recipients. Prudent selection of donors and adult recipients for SLT may expand the liver graft pool for pediatric patients without affecting outcomes in adults undergoing SLT.

Corresponding author: Shin Hwang

E-mail: shwang@amc.seoul.kr 